\title{
EL USO DEL AMBIENTE VIRTUAL CREPHIMAT PARA PROMOVER LA HISTORIA EN LA ENSEÑANZA DE LA MATEMÁTICA
}

\author{
Luis Andrés Castillo B. ${ }^{1}$ \\ luiscastleb@gmail.com \\ Iran Abreu Mendes ${ }^{1}$ \\ iamendes@gmail.com \\ ${ }^{1}$ Universidade Federal do Pará
}

Recibido: 29/10/2019 Aceptado: 15/01/2020

\section{Resumen}

Este artículo describe la experiencia de materialización del ambiente virtual interactivo denominado Centro Brasileño de Referencia en Pesquisas sobre Historia de las Matemáticas CREPHIMat. Experiencia que se origina a resultados parciales de un trabajo de maestría, vinculado al cumplimiento de objetivos específicos de dos macro proyectos, el primero intitulado: História para o Ensino da Matemática na Formação de Professores e na Educação Básica: uma Análise da Produção Brasileira (1997 - 2018), el segundo 1lamado: Uma história das pesquisas em História da Matemática no Brasil: produções, disseminações e contribuições à formação de professores de Matemática. Dichos proyectos son financiados por el Conselho Nacional de Desenvolvimento Científico e Tecnológico (CNPq) y sobre la coordinación del Prof. Dr. Iran Abreu Mendes. Algunos de los resultados obtenidos se refieren a la constitución de un gran acervo de producciones académico-científicas originadas en Brasil que traten sobre la historia de la matemática para el periodo de 1990-2018, unos 2000 archivos, entre Tesis, Disertaciones, artículos de revistas, memorias de congresos, libros de minicursos, productos educativos, materiales didácticos y demás producciones dirigidos a estudiantes, profesores y pesquisadores interesados en la historia de las matemáticas. Ademas se destacas algunas métricas y evaluaciones parciales del impacto que ha tenido el CREPHIMat desde su lanzamiento en agosto del 2019.

Palabras clave: CREPHIMat, ambientes virtuales, Historia de las Matematicas, repositorio.

\section{THE USE OF THE VIRTUAL CREPHIMAT ENVIRONMENT TO PROMOTE HISTORY IN THE TEACHING OF MATHEMATICS}

\begin{abstract}
This article describes the experience of materialization of the interactive virtual environment called Brazilian Reference Center for Research on the History of Mathematics - CREPHIMat. Experience that originates from partial results of a master's work, linked to the fulfillment of specific objectives of two macro projects, the first titled: Historia para o Ensino da Matemática na Formação de Professores e na Educação Básica: uma Análise da Produção Brasileira (1997 - 2018), the second called: Uma história das pesquisas em História da Matemática no Brasil: produções, disseminações e contribuições à formação de professores de Matemática. These projects are financed by the Conselho Nacional de Desenvolvimento Científico e Tecnológico $(\mathrm{CNPq})$ and on the coordination of Prof. Dr. Iran Abreu Mendes. Some of the results obtained refer to the constitution of a great collection of academic-scientific productions originated in
\end{abstract}


Brazil that deal with the history of mathematics for the period 1990-2018, about 2000 archives, including Theses, Dissertations, magazine articles, congress reports, books of mini-courses, educational products, didactic materials and other productions addressed to students, teachers and researchers interested in the history of mathematics. It also highlights some metrics and partial evaluations of the impact that CREPHIMat has had since its launch in August 2019.

Keywords: CREPHIMat, virtual environments, History of Mathematics, repository.

\title{
A UTILIZAÇÃO DO AMBIENTE VIRTUAL DO CREPHIMAT PARA PROMOVER A HISTÓRIA NO ENSINO DA MATEMÁTICA
}

\begin{abstract}
Resumo
Este artigo descreve a experiência de materialização do ambiente virtual interativo denominado Centro Brasileiro de Referência para Pesquisa em História da Matemática - CREPHIMat. Experiência oriunda de resultados parciais de um trabalho de mestrado, vinculada ao cumprimento de objetivos específicos de dois projetos macro, o primeiro intitulado: Historia para o Ensino da Matemática na Formação de Professores e na Educação Básica: uma Análise da Produção Brasileira (1997 - 2018), o segundo intitulado: Uma história das pesquisas em História da Matemática no Brasil: produções, disseminações e contribuições à formação de professores de Matemática. Esses projetos são financiados pelo Conselho Nacional de Desenvolvimento Científico e Tecnológico (CNPq) e sob a coordenação do Prof. Dr. Iran Abreu Mendes. Alguns dos resultados obtidos referem-se à constituição de uma grande coleção de produções acadêmico-científicas originárias do Brasil que tratam da história da matemática para o período 1990-2018, cerca de 2000 arquivos, incluindo teses, dissertações, artigos de revistas, relatórios de congressos, livros de mini-cursos, produtos educacionais, materiais didáticos e outras produções dirigidas a estudantes, professores e pesquisadores interessados em história da matemática. Também destaca algumas métricas e avaliações parciais do impacto que a CREPHIMat tem tido desde o seu lançamento em agosto de 2019.
\end{abstract}

Palavras chave: CREPHIMat, Ambientes virtuais, História da Matemática, repositorio.

\section{CONSIDERACIONES INICIALES}

En este artículo se describen los resultados parciales de la pesquisa en fase de desarrollo del trabajo de maestría del primero autor. Tal trabajo se inscribe en dos macro proyectos ${ }^{1}$ de investigación producidos y coordinados por el Prof. Dr. Iran Abreu Mendes y tutor académico del primer autor. Para posicionar los resultados obtenidos describiremos de manera sintetizada ambos proyectos. El primero se intitula: História para o Ensino da Matemática na Formação de Professores e na Educação Básica: uma Análise da Produção Brasileira (1997 - 2018), con este se pretende investigar las formas, significados y modalidades de abordar las producciones académicas que tratan de Historia de las Matemáticas y sus propuestas para su uso didáctico en

\footnotetext{
1 Proyectos certificados y financiados por el Conselho Nacional de Desenvolvimento Cientifico e Tecnológico (CNPq), Brasil.
} 
las clases de Matemáticas. El propósito central de este primer proyecto es responder a las siguientes cuestiones ¿Cómo las de tesis y disertaciones en Historia para la enseñanza de las Matemáticas son utilizadas por los profesores de Matemáticas en las escuelas públicas de Educación Básica? ¿Cómo reciben los profesores las producciones resultantes de estos estudios? ¿Las propuestas metodológicas para la enseñanza de las matemáticas basadas en información histórica, están incluidas en los libros de texto adoptados en las escuelas de Brasil?

En cuanto al segundo proyecto, fue intitulado: Uma história das pesquisas em História da Matemática no Brasil: produções, disseminações e contribuições à formação de professores de Matemática, en este se pretende describir y analizar cómo se configura el escenario histórico, epistemológico, pedagógico y patrimonial de la Historia de las Matemáticas en Brasil de 1990 a 2018. El análisis de la producción generada en la investigación en esta área de conocimiento permitirá señalar sus posibilidades de uso en la enseñanza, formación y acción de los profesores.

Estos proyectos surgen en respuesta al hecho de que hay una cantidad de producción académico-científica de historia de las matemáticas que no está llegando a las manos de los profesores de matemáticas de Educación Básica, o incluso de Educación Superior. Esta situación fue develada a partir de una investigación con una continuidad de tres décadas realizada por Mendes (Mendes, 2008, 2010, 2012, 2015, 2018a, 2018b) con el apoyo financiero del CNPq. Esta situación que evidencia un gran abismo que separa a los profesores de las producciones y de más materiales académicos es muy preocupante, ya que en las últimas décadas del siglo XX y las primeras del siglo XXI, la investigación en la historia de las matemáticas ha generado importantes contribuciones a la enseñanza de las matemáticas, destacando su potencial didáctico para apoyar la acción pedagógica de los profesores en la enseñanza de las matemáticas en todos los niveles educativos.

Para atender a las cuestiones anteriores Mendes (Mendes, 2018a, 2018b) planteo en estos proyectos los siguientes objetivos: (i) Planificar y organizar un inventario de la producción de tesis, disertaciones, libros, artículos, memorias de congresos, materiales didácticos y otras producciones didácticas para la enseñanza de las Matemáticas basadas y caracterizadas por el uso de informaciones sobre la históricas para apoyar la labor docente en las clases de Matemáticas, (ii) Clasificar y agrupar todos los materiales recolectados para componer un inventario temático organizado, de acuerdo con las tendencias enfatizadas en el material producido, (iii) Analizar las producciones sobre historia para la enseñanza de las Matemáticas, 
generadas en los grupos de investigación en Brasil que se ocupan de este tema de investigación, (iv) Describir los modelos teóricos surgidos de la investigación en historia para la enseñanza de las matemáticas que se centran directamente en la formación didáctica del profesor de matemáticas, y (v) Organizar el material digital a partir de las investigaciones realizadas y ponerlo a disposición de la comunidad académica en un espacio virtual de investigación e interacción.

Con los dos primeros objetivos se pretende recopilar un inventario de producciones académico-científicas de Historia de las Matemáticas en Brasil en el periodo de 1990-2018, con los siguientes dos se analizará y discutirá las producciones sobre historia para la enseñanza de las Matemáticas de modo que se puedan describir los modelos teóricos surgidos de la investigación que ofrezcan contribuciones para la enseñanza de la matemática y, finalmente, con el último objetivo se pretende material un local virtual en internet en forma de página web con el fin de difundir y diseminar el inventario temático de todas las producciones, de modo que los profesores de los diferentes niveles educativo puedan tener una vía que les permita conocer y explorar las contribuciones que estos diversos materiales tienen para apoyar su acción pedagógica en las clases de matemática.

Este espacio virtual fue concebido por Mendes (2018a) como el Centro Brasileño de Referencia en Pesquisas en Historia de las Matemáticas (CREPHIMat), un ambiente que además de tener la función de un repositorio digital de preservar, difundir y diseminar el inventario temático, también fue pensado para orientar y guiar a profesores en formación y en ejercicio, que el uso de informaciones histórica en las clases de Matemáticas, pueden en gran medida contribuir al logro de una enseñanza y aprendizaje significativo de las matemáticas. Además de lo anterior, se prevé que el CREPHIMat se constituya como un lugar de encuentro entre profesores e investigadores interesados en la historia de las matemáticas, que pueda ofrecer un espacio de formación para profesores de matemáticas de enseñanza primaria, secundaria y superior; y un espacio de apoyo para estudiantes de pregrado y posgrado en las áreas de matemática y enseñanza de la misma.

Con el objetivo de contribuir a una exploración didáctica y conceptual de las contribuciones de aproximadamente tres décadas de pesquisas en historia de las matemáticas, se materializo el CREPHIMat ${ }^{2}$. En este artículo se relata parte de la experiencia del primer autor

\footnotetext{
${ }^{2}$ Para acceder al ambiente virtual puede hacer clic aquí www.crephimat.com
} 
en la materialización del centro virtual, como parte de su trabajo de maestría desenvuelto en el Programa de Posgrado en Educación en Ciencias y Matemáticas de la Universidad Federal de Pará, Brasil.

En síntesis, se pretende informar sobre esta experiencia, seguido de la descripción del proceso y organización del entorno virtual, también discutir las potencialidades del CREPHIMat y finalmente, presentar algunas métricas que permiten inferir la aceptación en la comunidad que lo circunda. En la siguiente sección se presenta el ambiente virtual, constitución del mismo y el inventario temático.

\section{CENTRO BRASILEÑO DE REFERENCIA EN PESQUISAS EN HISTORIA DE LAS MATEMÁTICAS (CREPHIMat)}

El diseño, funcionalidades y constitución del Centro Brasileño de Referencia en Pesquisas en Historia de las Matemáticas, fue inspirado en dos ambientes virtuales oriundos de Brasil, el primero de éstos es el Centro de Referencia de Modelización Matemática en la Enseñanza (CREMM), un centro de Estudio e Investigación fundado por la Profa. Dra. Maria Salett Biembengut, en este centro el equipo del CREMM coloca a disposición un Sistema de Documentación (ver Figura 1) referente a investigaciones y a prácticas pedagógicas de Modelización Matemática en la Enseñanza de los más diversos países que puedan subsidiar alumnos, profesores e investigadores (CREMM, 2006).

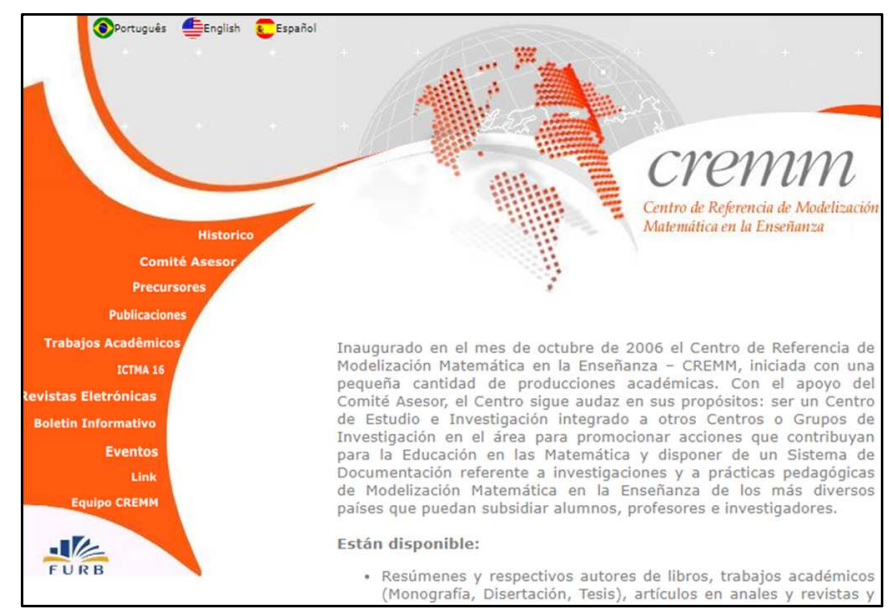

Figura 1. Página inicial del sistema de documentación del CREMM Fuente: http://www.furb.br/cremm/espanhol/index.php

En el repositorio que el CREMM pone a disposición se encuentran los resúmenes y respectivos autores de libros, trabajos académicos (Monografía, Disertación, Tesis), artículos en memorias de congreso y de revistas y experiencias pedagógicas, como también, tienen una 
atención por medio de la página en Internet, para orientar a alumnos, profesores e investigadores sea para la enseñanza como para la investigación.

De la influencia del CREMM, se decidió que la materialización del CREPHIMat fuese e formato de página web, que se localizara en los servidores de la Universidad Federal de Pará, del mismo modo como el CREMM está enlazado con la universidad que arropa a este centro. Sin embargo, por cuestiones de agilizar el proceso de elaboración y puesta en marcha del ambiente se decidió optar contratar un hospedaje y dominio privado. En cuestión a la navegación y organización de las secciones del CREPHIMat, homologamos el menú lateral del CREMM, adaptándolo a los contenidos e inventario temático propio del CREPHIMat como se puede ver en la Figura 2.

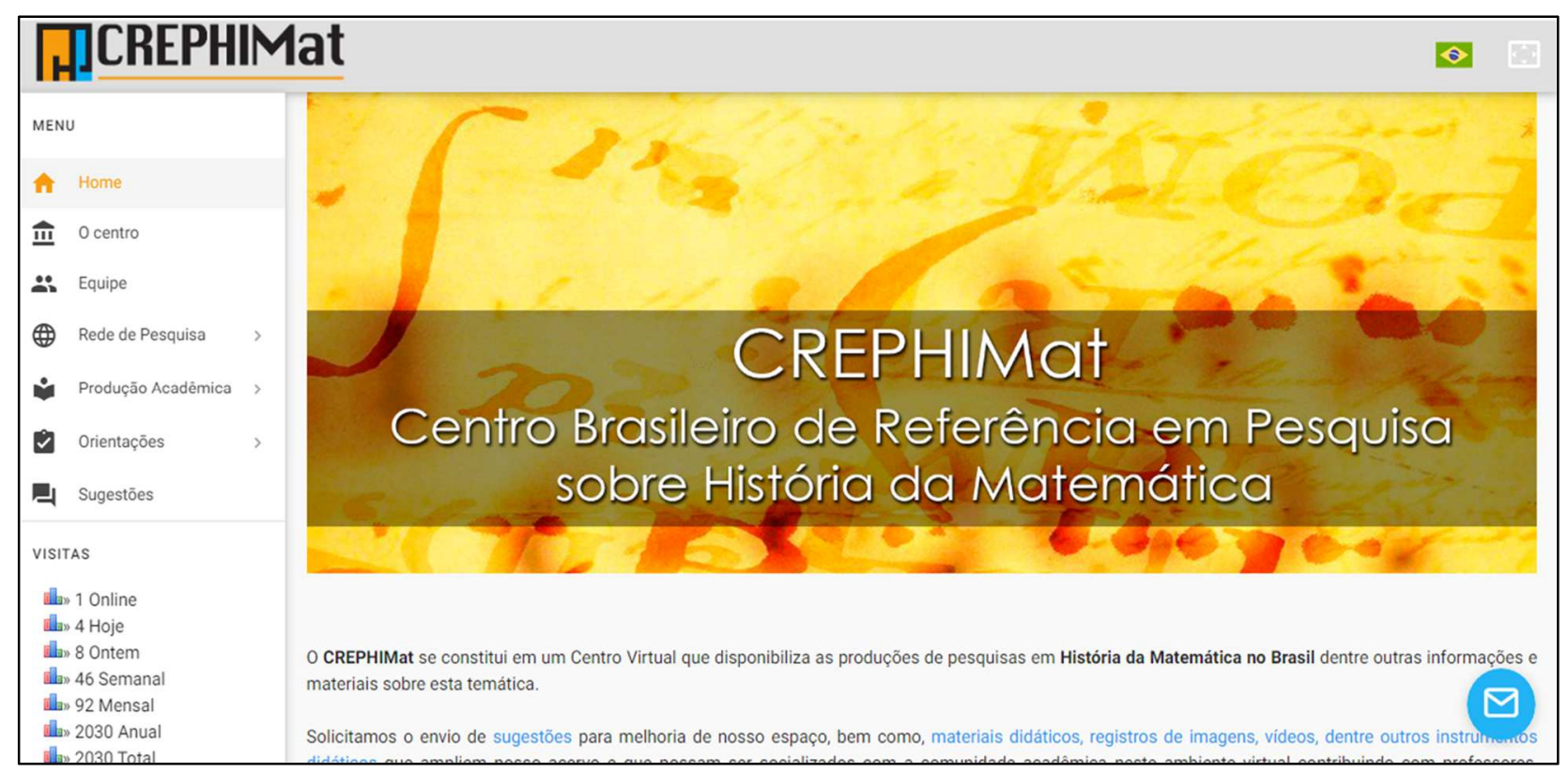

Figura 2. Página inicial del CREPHIMat

Fuente: http://www.crephimat.com/

El diseño del CREPHIMat fue basado en las tendencias visuales y estéticas del momento que son similares al patrón que siguen los aplicativos móviles de modo que fuera atractivo y fácil de navegación para tanto los usuarios nativos de esta humanidad digital como los que son más contemporáneas a las ultimas décadas del siglo XX. Una ventaja de este diseño tecnológico digital en el CREPHIMat, que lo diferencia del CREMM, es que tiene el ambiente virtual tiene el potencial de modificar dinámicamente los elementos contemplados en su diseño, de tal modo que se ajuste a las dimensiones de la pantalla del dispositivo por el cual es accesado. Un ejemplo de esto se puede observar en la Figura 3, en la cual el CREPHIMat visualizado 
desde un celular inteligente (o Smartphone, por su nombre en inglés), otro ejemplo se presenta en la Figura 4, en la cual se observa la forma como un usuario visualiza el ambiente virtual desde una Tablet, estas funcionalidades del CREPHIMat hacen posible brindar a nuestra comunidad una interacción con el ambiente virtual y con el inventario temático desde cualquier dispositivo y en cualquier localidad con acceso a internet.

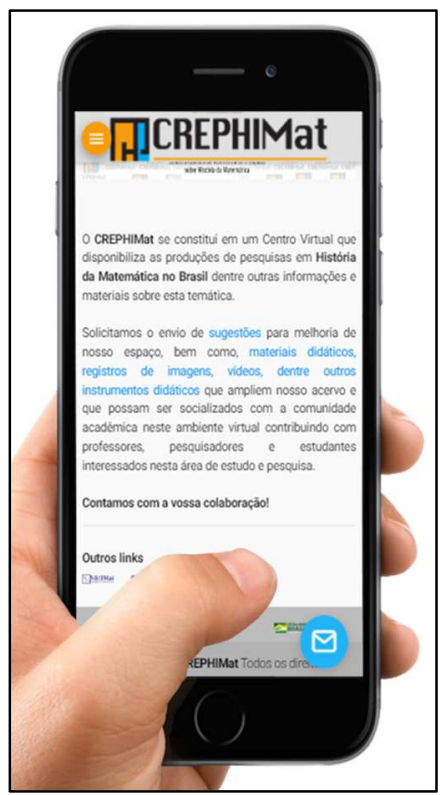

Figura 3. Página inicial del CREPHIMat desde Smartphone

Fuente: Elaboración propia de los autores

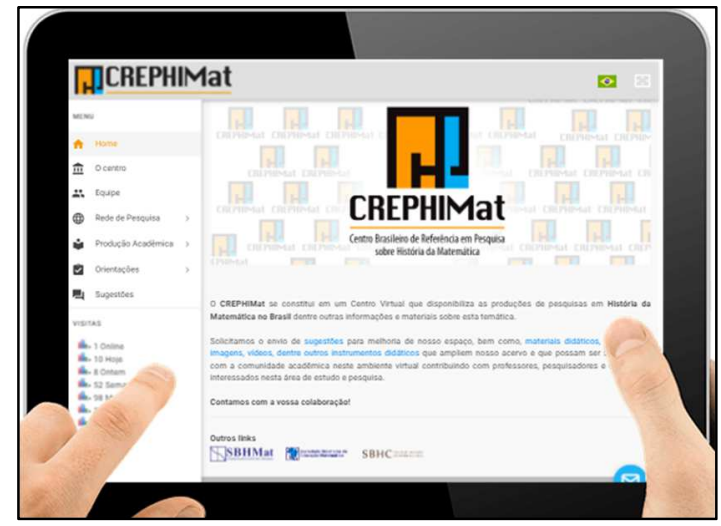

Figura 4. Página inicial del CREPHIMat desde una Tablet

Fuente: Elaboración propia de los autores

A pesar de que el CREMM su propio inventario temático, las informaciones disponibles de estas producciones eran especie de fichas con los datos de los autores, resumen, año de publicación, entre otras. Sin embargo, con el CREPHIMat se pretendía que además de disponibilidad estas informaciones de las producciones académicas también se colocasen a 
disposición el trabajo mismo. Entonces en nuestra búsqueda de un ambiente que nos diera un norte de cómo proceder en la fase de la constitución del CREPHIMat también como un repositorio digital, fuimos inspirados por el repositorio de contenido digital de la Universidad Federal de Santa Catarina (UFSC), repositorio desarrollado bajo el software libre DSpace ${ }^{3}$.

Llegamos a este ambiente por medio de una sub-comunidad en tal repositorio denominada História da Educação Matemática (l'Histoire de l'éducation mathématique) (ver Figura 5) administrada del Grupo de Pesquisa de História da Educação Matemática no Brasil (GHEMAT) y que contempla un inventario temático para pesquisadores con interés en la Historia de la Educación Matemática (Costa \& Valente, 2015). En las próximas secciones se comentarán las funcionalidades de este repositorio que fueron adoptadas para organizar y disponibilidad el inventario temático en el CREPHIMat.

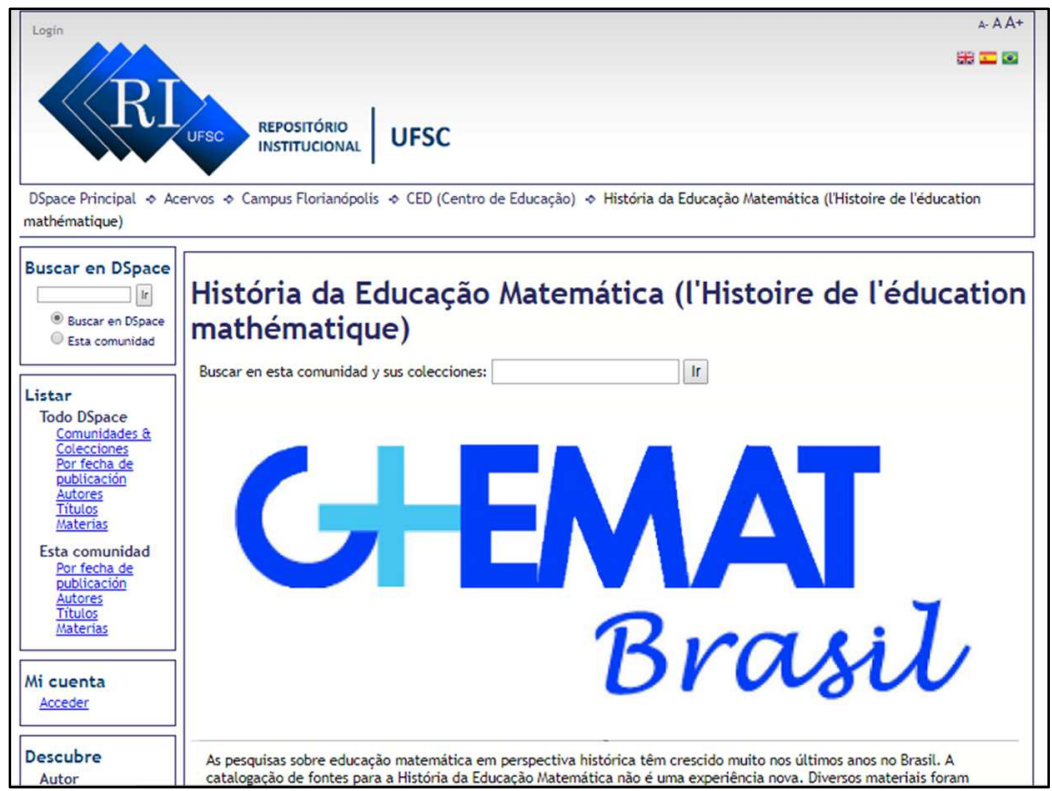

Figura 5. Página inicial del repositorio del GHEMAT

Fuente: https://repositorio.ufsc.br/handle/123456789/1769

Para este momento en la Figura ${ }^{4} 6$ presentamos la estructura de las secciones que componente al CREPHIMat, nos referimos de esta manera porque el CREPHIMat está pensado para ser un ambiente virtual dinámico en constante actualización y mejoramiento del mismo día a día por medio del equipo de trabajo y por sugerencias externas de la comunidad que lo

${ }^{3}$ Este software libre fue desarrollado para la administración y gestión de repositorios digitales, creando en cooperación entre el Instituto Tecnológico de Massachusetts - MIT y la Hewlett Packard Corporation. Para información más detalladas ver Costa \& Arruda (Costa \& Arruda, 2012)

${ }^{4}$ El idioma usado para representar las secciones del CREPHIMat en la figura 6 es portugués, el cual es el idioma original en el cual se presentan todas las informaciones en dicho centro virtual. 
frecuenta. Las primeras tres secciones llamadas: Home, O Centro y Equipe describen en líneas generales la constitución de centro, sus objetivos, contenidos, y quienes laboran en el ambiente para que esté disponible al mundo por medio de la internet.

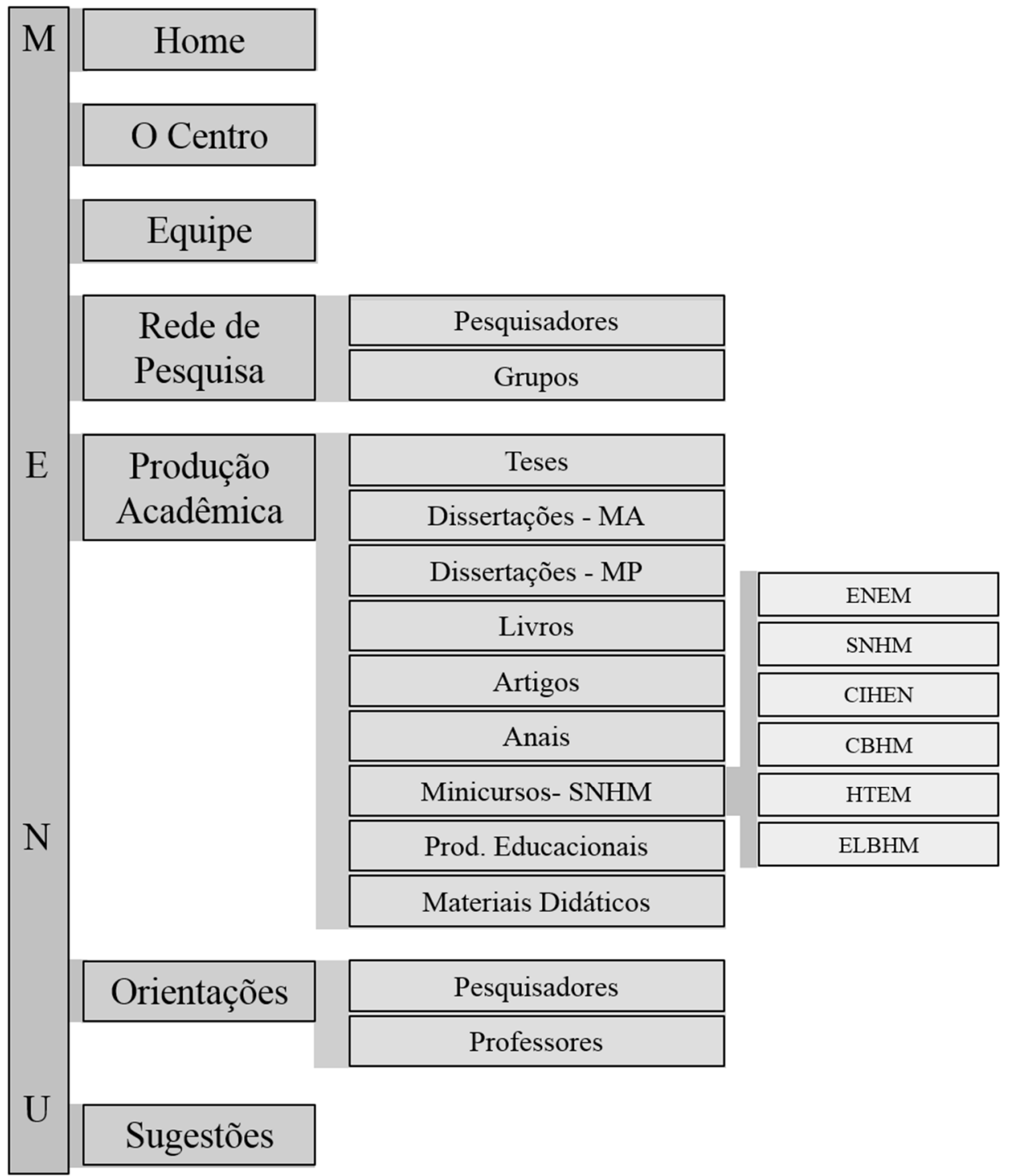

Figura 6. Estructura de las secciones del CREPHIMat

Fuente: Elaboración propia de los autores

La sección Rede de pesquisa presenta dos inventario temático; el primero de estos de profesores e investigadores del área de historia de la matemática y/o con alguna relación a esta, el segundo presenta un inventario de grupos de pesquisas dividíos en dos categorías: la primera contempla a todos los grupos cuyo foco es la historia de la matemática y/o educación matemática, la segunda se constituyó por todos aquellos grupos donde la historia de la matemática y/o educación matemática figura como una de sus líneas de investigación. 
La siguiente sección Produção Acadêmica es el, anatómicamente hablando, corazón del CREPHIMat. Dado que en esta parte del centro se encuentra organizado y disponible un gran inventario temático de (por lo menos) 2000 producciones académicas que contemplan Artículos de revistas, memorias de congresos, materiales didácticos, libros de Minicursos, tesis, disertaciones y productos educacionales que se derivan disertaciones de maestrías profesionales, vale destacar que estos últimos tres se encuentran organizados según las tendencias emergentes de la investigación en Historia de las Matemáticas identificadas en los resultados obtenidos en 10 años de investigación en esta área, según Mendes (2010, 2015) estas son:

Historia y Epistemología de las Matemáticas - HEpM, se refiere a las producciones científico-académicas que se relacionan tanto con la vida como con el trabajo de los matemáticos, así como con el desarrollo de sus ideas matemáticas y el desarrollo de conceptos matemáticos en el tiempo. Historia de la Educación Matemática - HEdM, cuyas producciones abordan estudios relacionados con la historia de las instituciones, (auto)biografías de profesores de matemáticas, además de las contribuciones realizadas por ellos para la formación de profesores de matemáticas y para la mejora de la enseñanza, así como lo que contribuyen a la colección de documentos, memorias y el patrimonio de la Educación Matemática. Historia para la Enseñanza de las Matemáticas - HEnM, cuyas producciones se caracterizan por propuestas y acciones centradas en los usos de la información histórica con fines pedagógicos, como estrategia para la enseñanza de las matemáticas, así como el desarrollo de materiales didácticos para la enseñanza de las matemáticas, basados en fuentes históricas.

Al respecto de la sección de Orientações, en esta se pretender ofrecer, por un lado, sugerencias prácticas sobre las producciones del inventario temáticos en el CREPHIMat y del modo de cómo usar el mismo para que los profesores trasciendan sus dificultades conceptuales y didácticas. Por el otro lado, sugerencias a Pesquisadores sobre las cuestiones en abierto que las tesis y disertaciones tienen para iniciar o continuar pesquisas inéditas sobre la historia de las matemáticas y/o Educación Matemática. Por el momento esta sección se encuentra en construcción. Por ultimo en nuestro orden, se encuentra la sección, Sugestões en la cual es una primera vía de comunicación disponible entre la comunidad que accede al CREPHIMat y su equipo de trabajo. En el siguiente apartado, se presenta y describe que ofrece el CREPHIMat para los profesores con interés de usar la historia de la matemática en su acción docente.

\section{PRODUCCIONES ACADÉMICAS EN EL CREPHIMAT}


Antes de comenzar a describir el inventario ${ }^{5}$ temático de las producciones que el CREPHIMat ofrece a los profesores para apoyar su acción pedagógica en las aulas de matemática, queremos destacar a pesar que se cuentan con producciones que están encajadas en la tendencia de Historia para la Enseñanza de las Matemáticas, se tienen investigaciones que destacan las potencialidades didácticas y conceptuales que las producciones en las otras tendencias de HEpM y HEdM poseen para la movilización de contenidos matemáticos en las aulas (Barros \& Mendes, 2017, 2019; Gonçalves \& Mendes, 2015).

Las primeras producciones en el entorno virtual disponibles para ser consultadas por los profesores, investigadores y profesores en formación inicial, es la colección de Tesis y Disertaciones las cuales fueron recopiladas desde su primera búsqueda realizada por medio del proyecto intitulado: Cartografias da produção em História da Matemática no Brasil: um estudo centrado nas dissertações e teses defendidas entre 1990-2010, coordinado por el Prof. Dr. Iran Abreu Mendes, tal acervo fue ampliado con la ejecución de los proyectos antes mencionados hasta abarcar un periodo de tres décadas, es decir, desde 1990 hasta 2018. Con este levantamiento de producciones so obtuvo un total setecientas y cincuenta y siete (757) entre tesis y disertaciones (tanto de maestrías académicas como profesionales), en el siguiente cuadro 1 pueden observarse la distribución de estos trabajos según su nivel y tendencia de pesquisa:

Cuadro 1. Tesis y Disertaciones de Historia de la Matemática en Brasil: Niveles y Tendencias

\begin{tabular}{|c|c|c|c|c|}
\hline Nivel & HEpM & HEdM & HEnM & Total \\
\hline Doctorado & 78 & 143 & 19 & 240 \\
\hline Maestría Académica & 57 & 273 & 57 & 387 \\
\hline Maestría Profesional & 10 & 47 & 73 & 130 \\
\hline Total & 145 & 467 & 149 & 757 \\
\hline
\end{tabular}

Fuente: Mendes (2019)

\footnotetext{
${ }^{5}$ Este gran acervo de producciones ha sido constituido por un trabajo en cooperación entre el grupo de orientados y becarios de iniciación científica dirigidos por el coordinador de los proyectos antes mencionados, para más detalles de los involucrados y de los proyectos vinculados puede visitar el currículo lattes Prof. Iran Abreu Mendes: http://lattes.cnpq.br/4490674057492872
} 
En el CREPHIMat se disponibilizan estas producciones separadas por secciones según su el tipo de documento: Tesis, Disertaciones de Maestrías Academias (MA) y Disertaciones de Maestrías Profesionales (MP), y a su vez cada una de estas se subdivide según las tendencias de pesquisa: HEpM, HEdM, HenM (ver Figura 7).

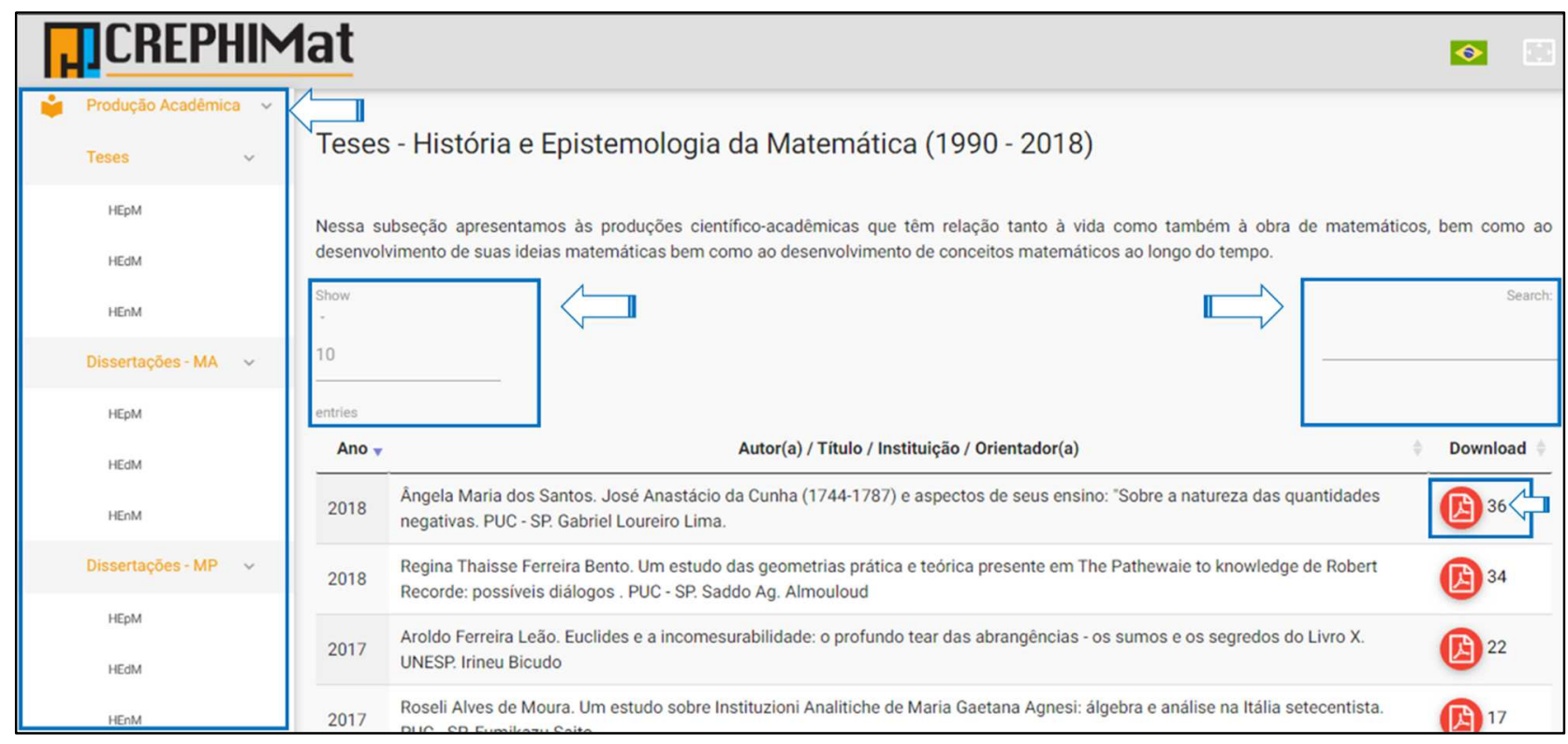

Figura 7. Organización de Tesis y Disertaciones en el CREPHIMat

Fuente: Elaboración propia de los autores

En la Figura 7 podemos observar la subsección dedicada a las tesis catalogadas en la tendencia de HEpM, la cual se constituye por un título, una breve descripción de las características generales de las producciones en esta parte del CREPHIMat y seguidamente se presentan las producciones dispuesta en una tabla dinámica. Tabla realizada en el entorno DataTables $^{6}$ lo cual permite agregar controles de interacción avanzados y filtros que permite una búsqueda más dinámica con resultados instantáneos si tener que actualizar el contenido de la página.

Estos controles se pueden apreciar encima de la tabla, al lado izquierdo se encuentra el Show Entries, con el cual se pueden modificar el número de registro que se muestran en la tabla; el Search otro controlador que se localiza al lado contrario del primero, se utiliza para filtrar la búsqueda en las producciones según palabras claves que se inserten en este controlador, otro controlador es que este plugin crea una paginación en la parte inferior de la tabla para facilitar

${ }^{6}$ DataTables está disponible bajo la licencia MIT. Esto quiere decir, que es libre para ser utilizado DataTables como se desee, incluyendo la modificación y redistribución del código, siempre y cuando se conserve el aviso de copyright original. Para mayor información: https://datatables.net/manual/index 
la visualización de los registro. Destacamos que este diseño fue decidido como patrón para organizar y disponibilizar los otros tipos de producciones en el CREPHIMat.

Otra producción para ser consultada en el centro virtual son los artículos de revistas que tratan temas relacionados con la historia de las matemáticas y/o educación matemática. Esta sección se componen de artículos buscados y catalogados de las siguientes revistas Brasilera: Boletim de Educação Matemática - BOLEMA, Revista Brasileira de História da Ciência RBHC, Zetetiké, Revista Brasileira de História da Matemática - RBHM, Revista de Matemática, Ensino e Cultura - REMATEC, Revista Eletrônica de Educação Matemática REVEMAT, COCAR, Alexandria: Revista de Educação em Ciência e Tecnologia, Revista História da Matemática para Professores - RHMP, Revista de História da Educação Matemática - HISTEMAT, HIPÁTIA: Revista Brasileira de História, Educação e Matemática.

Destacamos que la elección de estas revistas se justifica por su relación directa con la historia de las matemáticas y/o la Educación Matemática en su corpus, así como por la clasificación de las mismas en el Qualis Ensino en la plataforma Sucupira ${ }^{7}$. En el siguiente cuadro 2 presentamos la distribución de los artículos de cada revista según su periodo de origen hasta el 2018 y la cantidad de artículos que tratan temas relaciones con la historia de la matemática.

\footnotetext{
${ }^{7}$ Para mayor detalles de la plataforma sucupira: https://www.capes.gov.br/avaliacao/plataforma-sucupira
} 
Cuadro 2: Artículos de revistas Brasileiras de historia de la matemática (1985-2018)

\begin{tabular}{|c|c|c|c|}
\hline Revista & $\begin{array}{c}\text { Número de } \\
\text { artículos }\end{array}$ & $\begin{array}{c}\text { Historia de la Matemática } \\
\text { (HM) }\end{array}$ & Porcentaje \\
\hline BOLEMA $(1985-2018)$ & 772 & 74 & $10 \%$ \\
\hline RBHC (1985-2018) & 500 & 06 & $1 \%$ \\
\hline Zetetiké $(1993-2018)$ & 397 & 24 & $6 \%$ \\
\hline RBHM (2001-2017) & 216 & 152 & $70 \%$ \\
\hline REMATEC $(2006-2018)$ & 218 & 62 & $28 \%$ \\
\hline REVEMAT $(2006-2018)$ & 291 & 09 & $3 \%$ \\
\hline COCAR $(2007-2018)$ & 352 & 05 & $1 \%$ \\
\hline Alexandria $(2008-2018)$ & 322 & 04 & $68 \%$ \\
\hline RHMP (2013-2016) & 28 & 19 & $89 \%$ \\
\hline HISTEMAT (2015-2018) & 128 & 114 & $22 \%$ \\
\hline HIPATIA (2016-2018) & 27 & 06 & $15 \%$ \\
\hline TOTAL & 3251 & 475 & \\
\hline
\end{tabular}

Fuente: Castillo \& Mendes (2019)

En cuanto a las Memorias de Congreso, durante la pesquisa se han captado las producciones de seis (06) eventos científicos, el primero de éstos son las publicadas por el Encontro Nacional de Educação Matemática - ENEM (1987 - 2016), es el más importante evento a nivel nacional, porque reúne el universo de las tendencias en Educación Matemática y un diverso público de profesores de Educación Básica, profesores y alumnos de Licenciatura y Pedagogía Matemática, estudiantes de Postgrado e investigadores. En la celebración de cada encuentro se destaca el interés en las discusiones sobre la Educación Matemática, sus múltiples y complejas acciones, las tendencias metodológicas y la investigación que constituyen el área, entre estas la historia de las matemáticas. Actualmente se cuentan con las 10 ediciones pasada de las memorias del evento (Figura 8), en espera de su más reciente edición después de la conmoración del XIII ENEM en julio de este año. 


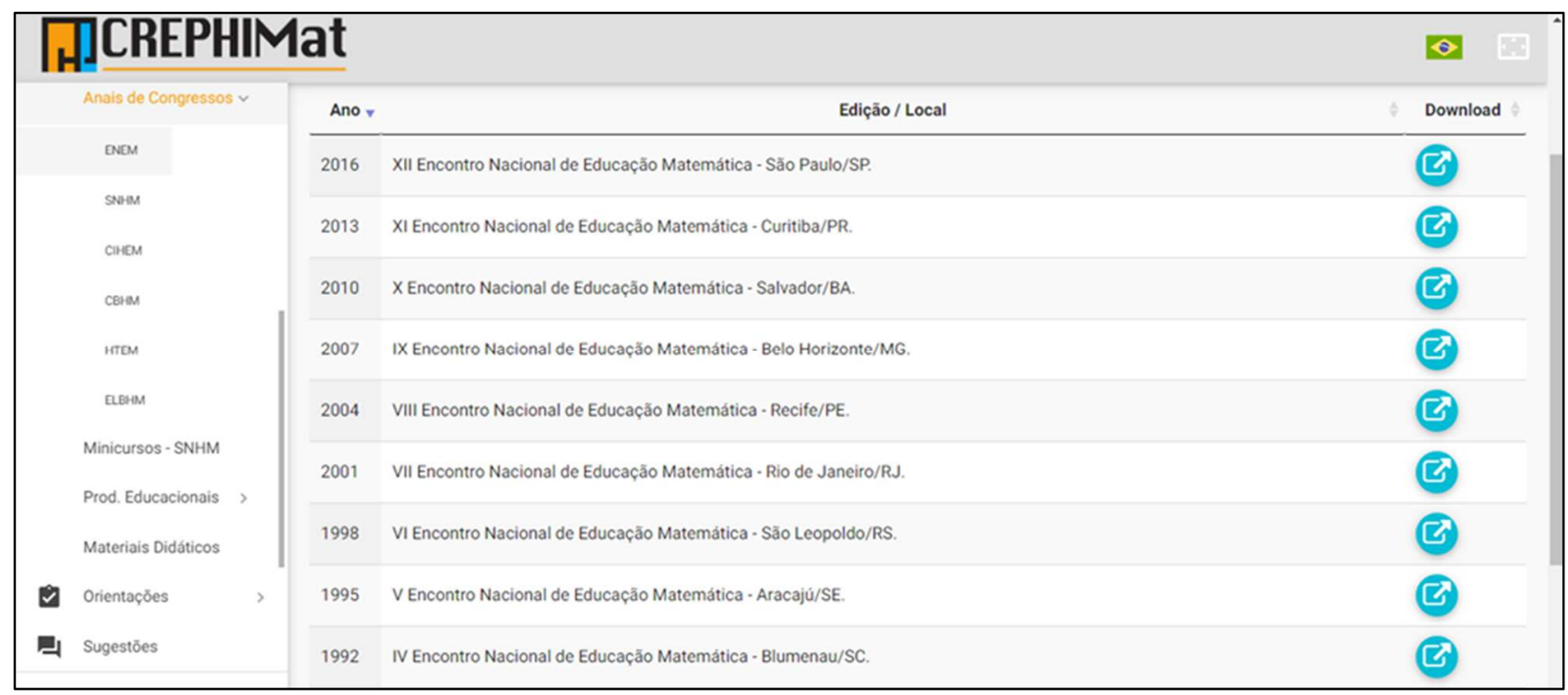

Figura 8. Memorias del ENEM en el CREPHIMat

Fuente: Elaboración propia de los autores a partir de http://www.crephimat.com/enem

En esta sección están disponibles las memorias del Seminário Nacional de História da Matemática - SNHM celebrados desde 1995, que se celebran cada dos años, siempre en los días previos a la Semana Santa. Los seminarios nacionales son una de las formas explícitas de alcanzar los objetivos estatutarios del SBHMat, caracterizados por un amplio programa de carácter científico y pedagógico en el que se presentan las nuevas producciones de conocimiento del área. Se debaten grandes temas, se exponen problemas en busca de soluciones, se difunden experiencias, bibliografías y materiales didácticos, con el objetivo de promover el desarrollo y la difusión de experiencias, estudios y reflexiones en el área de Historia de las Matemáticas.

Están disponibles las memorias de las trece (13) ediciones del evento (Figura 9) desde el I SNHM realizado en 1995 en Recife (PE), del II SNHM en Águas de São Pedro (SP) en 1997, del III SNHM en 1999 en Vitória en el estado de Espírito Santo, del IV SNHM en Natal (RN) ocurrido en 2001, del V SNHM en 2003 en Río Claro (SP), del VI SNHM en 2005 realizado en Brasilia (DF), del VII SNHM en Guarapuava (PR) en 2007, el VIII en Belém (PA) en 2009, el IX SNHM en 2011 en Aracaju (SE), el X SNHM en 2013 en Campinas (SP), el XI SNHM en 2015 en Natal (RN), el XII SNHM en 2017 en Itajubá (MG) y el más reciente, el XIII SNHM en Fortaleza (CE) en 2019. 


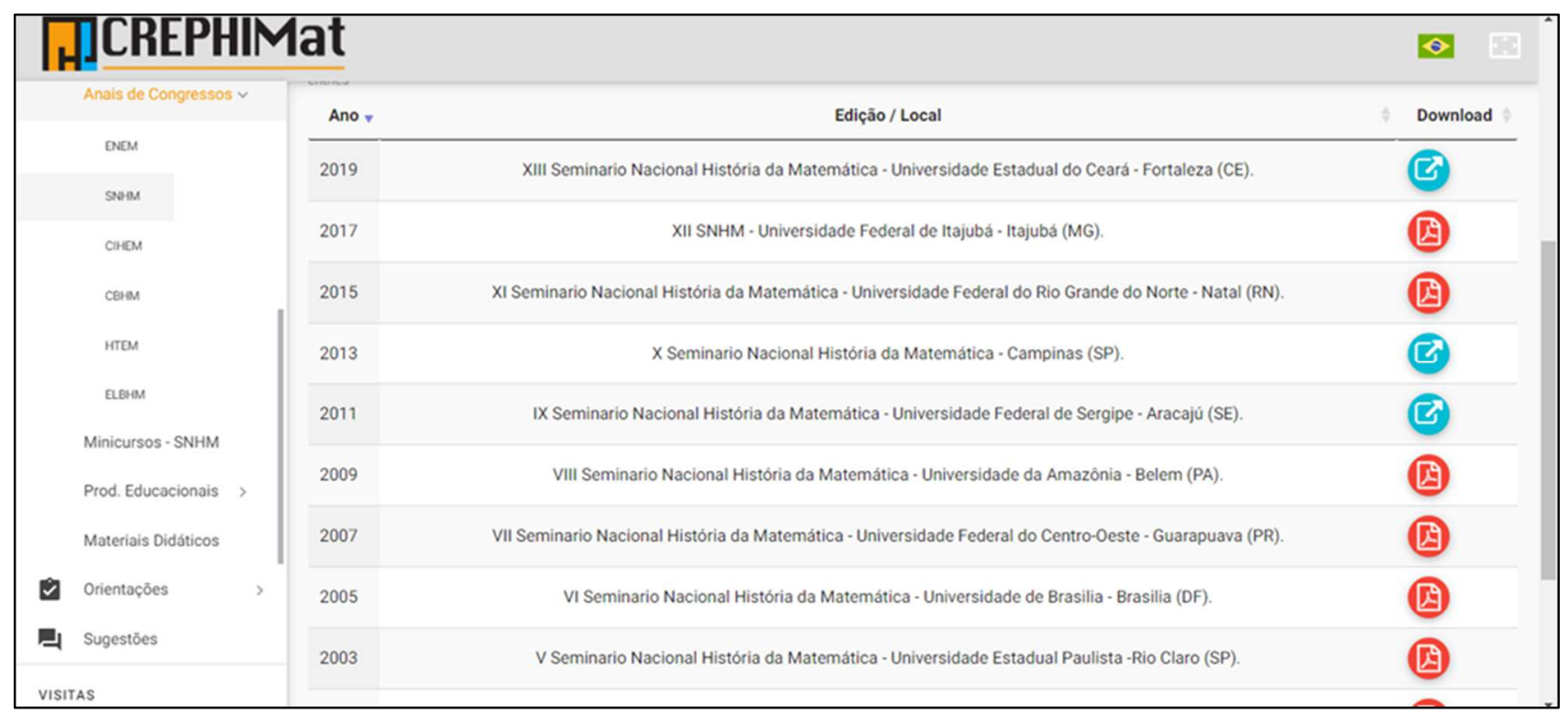

Figura 8. Memorias del SNHM en el CREPHIMat

Fuente: Elaboración propia de los autores a partir de http://www.crephimat.com/snhm

Las siguientes memorias disponibles en el CREPHIMat son del Congreso Iberoamericano de Historia de la Educación Matemática - CIHEM, el cual es un evento académico bianual, de carácter científico y tecnológico, que ha continuado la idea de consolidar una comunidad muy amplia y con diversidad de intereses. Es así como allí han convergido Historiadores, Educadores, Matemáticos, o una integración total o parcial de los anteriores, con la finalidad de divulgar resultados de investigación de alto impacto obtenidos por iniciativas institucionales o individuales de distintas universidades.

Los anteriores congresos fueron realizados en 2011 en Covilhã (Portugal); en 2013, en Cancún (México); en 2015, en Belém do Pará (Brasil); y el último en el 2017, Murcia (España), la ralización del evento en estos años se ha constituido en un escenario importante de divulgación y discusión de actividades y proyectos en este nuevo campo de estudios. Actualmente se incorporan en el CREPHIMat las cuatro memorias del evento (Figura 9). 


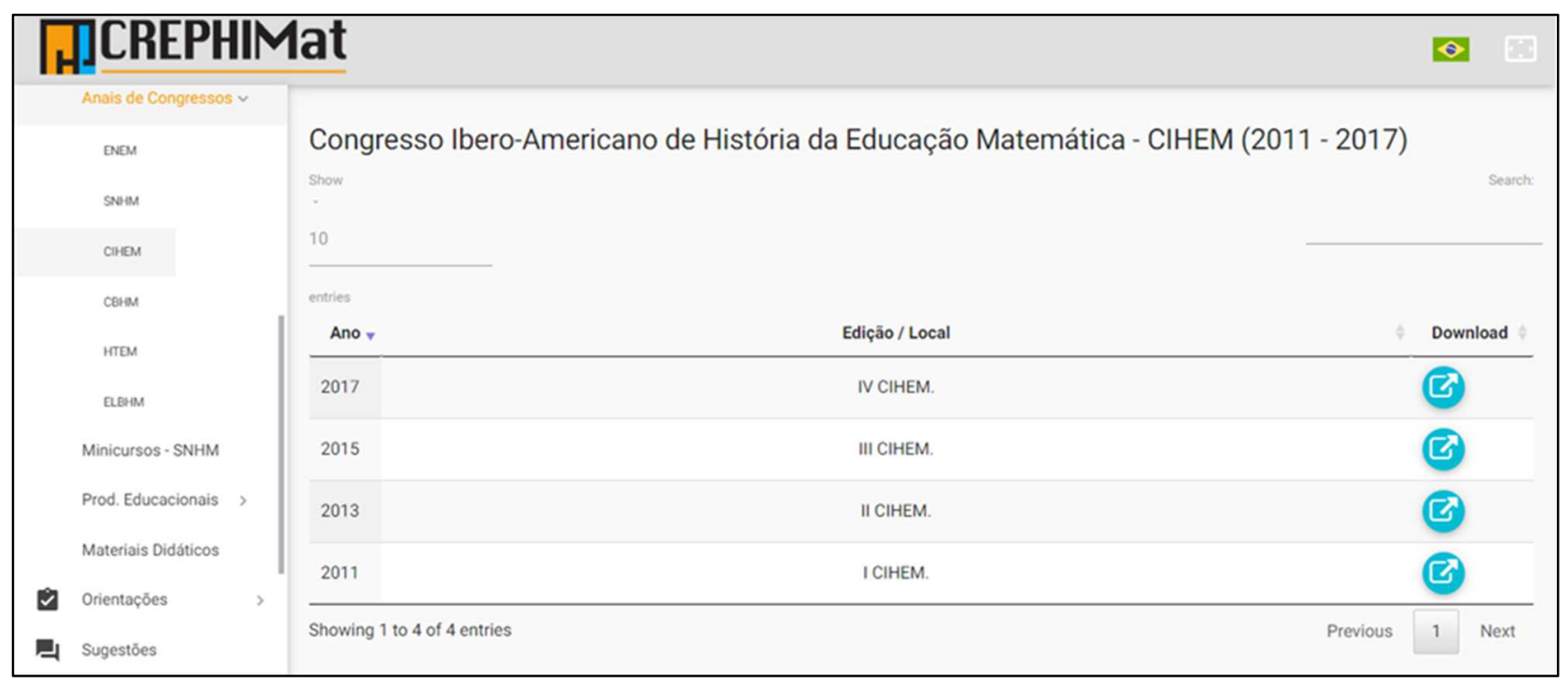

Figura 9. Memorias del CIHEM en el CREPHIMat

Fuente: Elaboración propia de los autores a partir de http://www.crephimat.com/cihem

Las siguientes memorias presentes en el CREPHIMat son del Colóquio de História e Tecnologia no Ensino de Matemática - HTEM, fue lanzado en 2002, con el objetivo de crear un espacio de discusión sobre las contribuciones y el impacto de la investigación en la historia de las matemáticas y sobre el papel de las tecnologías en la enseñanza de las matemáticas. Varios eventos tratan a veces de las Tecnologías en la Educación Matemática, a veces de la Historia de las Matemáticas, pero en general, hay pocas conexiones entre estos temas. La convicción del grupo de investigadores que encabezó la realización del HTEM es que el tratamiento articulado de los componentes Historia, Tecnología, Enseñanza y Matemáticas permite una lectura original y fructífera de los fenómenos relacionados con la adquisición y transmisión del conocimiento matemático.

El HTEM no está vinculado a una entidad específica, pero ha recibido apoyo institucional de varias asociaciones, tales como: Sociedad Brasileña de Educación Matemática (SBEM), Sociedad Brasileña de Matemáticas Aplicadas y Computacionales (SBMAC), Sociedad Brasileña de Matemáticas (SBM) y Sociedad Brasileña de Historia Matemática (SBHMat).

El I HTEM se celebró en febrero de 2002, en la UERJ, el II HTEM se celebró de nuevo en la UERJ, en marzo de 2004, el III HTEM se celebró en la PUC-SP en mayo de 2006, el IV HTEM se celebró en mayo de 2008, en la UFRJ fue promovido por el Programa de Postgrado en Enseñanza de Matemáticas de la UFRJ y por el Laboratorio de Investigación y Desarrollo en Enseñanza de las Matemáticas y las Ciencias (LIMC) de la UFRJ, Del 25 al 30 de julio de 2010 
se realizó en Recife, Brasil, el V HTEM, promovido por el Programa de Postgrado en Educación Matemática y Tecnológica (EDUMATEC) de la UFPE y organizado por el Grupo de Estudio sobre Nuevas Tecnologías y Educación. Finalmente, el VI HTEM se celebró en São Carlos, en 2013. Como se puede apreciar en la Figura 10, se disponen por el momento de las memorias del III y V HTEM.

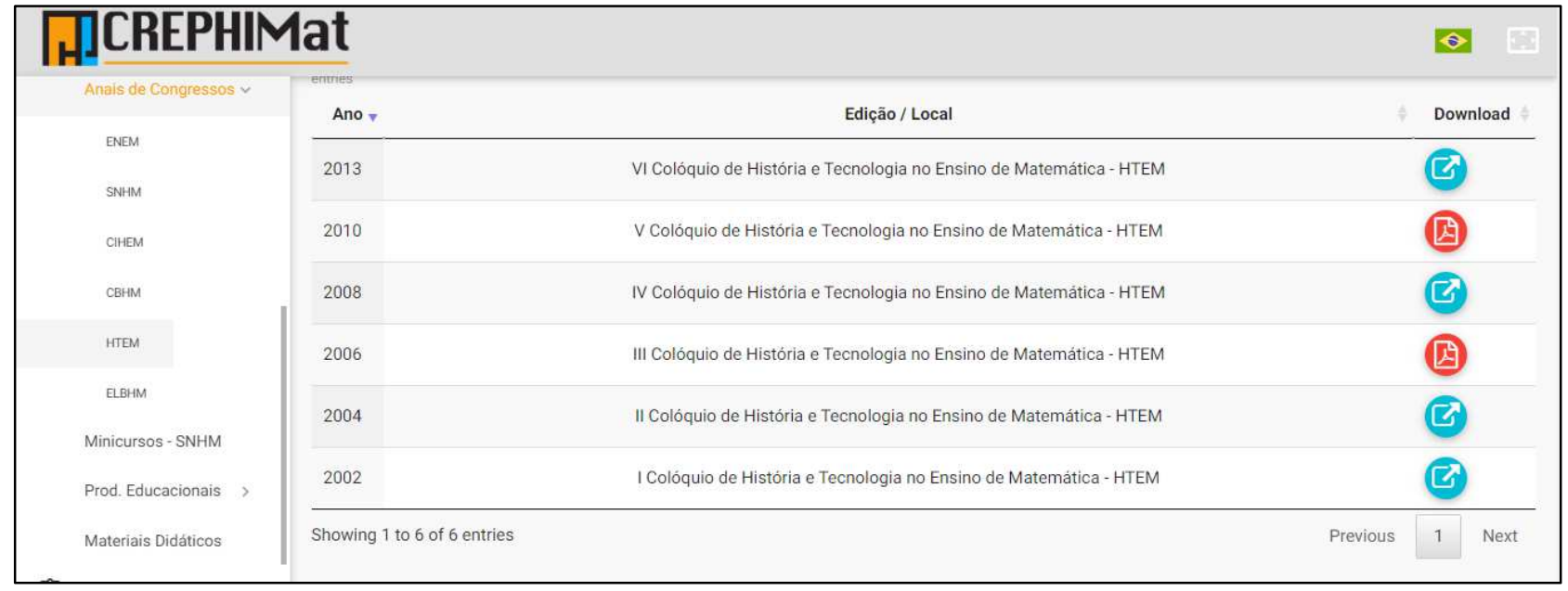

Figura 10. Memorias del HTEM en el CREPHIMat

Fuente: Elaboración propia de los autores a partir de http://www.crephimat.com/htem

Las siguientes memorias disponibles en el CREPHIMat son del Encontro LusoBrasileiro de História da Matemática es un evento internacional que reúne a investigadores e interesados en la Historia de las Matemáticas de Brasil y Portugal. La responsabilidad de su organización es una acción conjunta entre la Sociedade Brasileira de História da Matemática (Brasil) y el Seminário Nacional de História da Matemática (Portugal). Comenzando en Coimbra, Portugal, en 1993, su objetivo es fortalecer las relaciones científicas en esta área entre los investigadores de los dos países donde tienen lugar los eventos. Inicialmente, el evento fue pensado para ser organizado en reuniones cada 4 años. Hoy el algoritmo muda un poco, en el sentido de que cada Encuentro en Brasil tiene lugar 4 años después del anterior en Portugal, y que cada Encuentro en Portugal tiene lugar 3 años después del anterior en Brasil.

El $1^{\circ}$ ELBHM fue celebrado en la Universidad de Coimbra, Portugal, del 31 de agosto al 3 de septiembre de 1993, luego el $2^{\circ}$ ELBHM en Águas de São Pedro, São Paulo, Brasil, del 23 al 26 de marzo de 1997, después el $3^{\circ}$ ELBHM nuevamente en la Universidad de Coimbra, Portugal, del 7 al 12 de febrero de 2000. El 4 ELBHM dirigido por la Universidade Federal do Rio Grande do Norte, Natal, Brasil, 24-27 de octubre de 2004, el 5 ELBHM organizado por el 
Cine-Teatro Avenida e Biblioteca Municipal, Castelo Branco, Portugal, del 3 al 7 de octubre de 2007, el $6^{\circ}$ ELBHM en la Universidade Federal de São João Del-Rei, Minas Gerais, Brasil, del 28 al 31 de agosto de 2011, el $7^{\circ}$ ELBHM se celebró en las locaciones del Auditorio Municipal, Museo Abilio de Mattos e Silva y Museo Municipal, Óbidos, Portugal, del 15 al 19 de octubre de 2014 y finalmente su más reciente edición el $8^{\circ}$ ELBHM fue organizado por el Centro de Ingeniería y Ciencias Exactas de la Universidad Estatal del Oeste del Paraná, Parque Tecnológico Itaipú, Foz do Iguaçu, Paraná, Brasil, del 13 al 16 de agosto de 2018. De estas ediciones se cuentan con las memorias de sus últimas tres ediciones como se puede apreciar en la Figura 11.

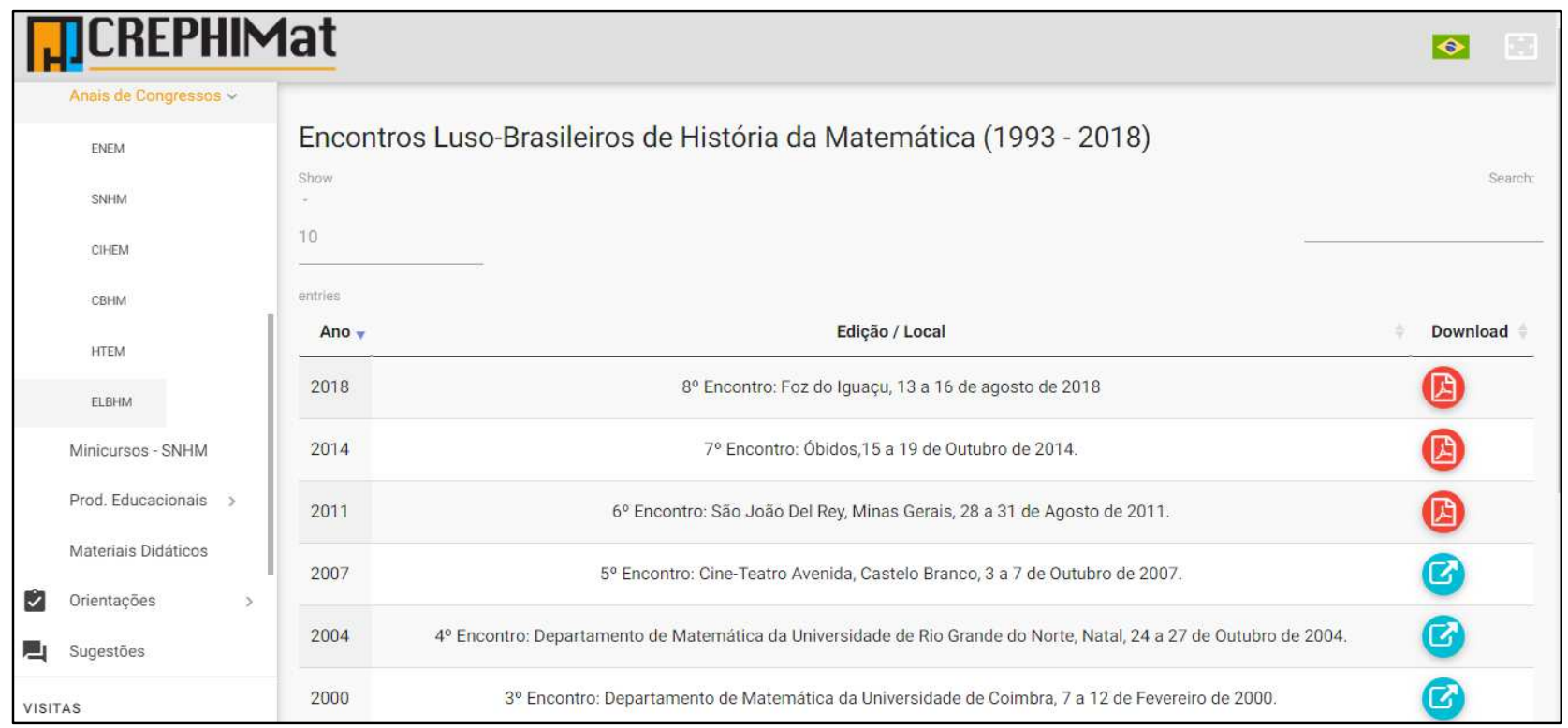

Figura 11. Memorias del ELBHM en el CREPHIMat

Fuente: Elaboración propia de los autores a partir de http://www.crephimat.com/elbhm

La siguiente producción que se contempla en el CREPHIMat son los libros sobre la historia de las matemáticas para uso didáctico de los profesores de matemáticas que se originó en el IV Seminário Nacional de História da Matematica (IV SNHM), realizado en Natal (Rio Grande do Norte), en 2001. En ese año se publicaron nueve títulos referidos a diversos temas. La receptividad de los textos, por parte de los alumnos de pregrado en matemáticas y de los profesores de los tres niveles de enseñanza (primaria, secundaria y superior), hizo que SBHMat llevara a cabo el proyecto, con el fin de contribuir a la difusión y utilización de esta producción en las clases de matemáticas de los distintos niveles de enseñanza. Desde el SNHM de 2001 al 2017, a lo largo de estas 12 ediciones del Evento, la Sociedad Brasileña de Historia de las Matemáticas (SBHMat) ha publicado un total de 101 libros de Minicursos. 
En el siguiente cuadro 3 se presenta la distribución los libros de Minicurso publicados desde su prime edición en 2001 hasta el 2017, estos clasificados según la tendencia de pesquisa de historia de la matemática y el porcentaje de cada total de libros por evento.

Cuadro 3: Libros de Minicursos del SNHM: Tendencias

\begin{tabular}{|c|c|c|c|c|c|c|c|}
\hline SNHM (2001 - 2017) & Libros & HEpM & \% & HEdM & \% & HEnM & $\%$ \\
\hline IV & 9 & 6 & $67 \%$ & 1 & $11 \%$ & 2 & $22 \%$ \\
\hline V & 11 & 6 & $54 \%$ & 2 & $18 \%$ & 3 & $28 \%$ \\
\hline VI & 11 & 3 & $27 \%$ & 1 & $9 \%$ & 7 & $64 \%$ \\
\hline VII & 12 & 3 & $25 \%$ & 5 & $42 \%$ & 4 & $33 \%$ \\
\hline VIII & 19 & 9 & $47 \%$ & 3 & $16 \%$ & 7 & $37 \%$ \\
\hline IX & 12 & 7 & $58 \%$ & - & - & 5 & $42 \%$ \\
\hline X & 7 & 4 & $57 \%$ & 3 & $43 \%$ & - & - \\
\hline XI & 10 & 1 & $10 \%$ & 3 & $30 \%$ & 6 & $60 \%$ \\
\hline XII & 10 & 3 & $30 \%$ & 1 & $10 \%$ & 6 & $60 \%$ \\
\hline Total & 101 & 42 & $41 \%$ & 19 & $19 \%$ & 40 & $40 \%$ \\
\hline
\end{tabular}

Fuente: Adaptado de Silva, Silva-Neto \& Castillo (2019)

Para presentar a los profesores un panorama de los temas matemáticos inherentes a los Minicursos que tratan las referidas dimensiones del cuadro 3, el siguiente cuadro 4 muestra en términos cuantitativos las producciones referidas a cada tópico matemático abordado por cada libro de los minicursos ofrecidos en los SNHM.

Cuadro 4: Libros de Minicursos del SNHM: Tópicos Matemáticos

\begin{tabular}{|c|c|c|c|c|c|c|}
\hline $\begin{array}{c}\text { SNHM } \\
(\mathbf{2 0 0 1}-\mathbf{2 0 1 7})\end{array}$ & Aritmética & Álgebra & Geometría & Trigonometría & Otros & Total \\
\hline IV & 2 & - & 5 & 1 & 1 & 9 \\
\hline V & 4 & 2 & 3 & - & 2 & 11 \\
\hline VI & 6 & - & 3 & 2 & - & 11 \\
\hline VII & 1 & 4 & 1 & - & 6 & 12 \\
\hline VIII & 2 & 4 & 7 & 2 & 4 & 19 \\
\hline IX & 2 & 3 & 6 & - & 1 & 12 \\
\hline X & 1 & 4 & 1 & - & 1 & 7 \\
\hline XI & 1 & 2 & 3 & - & 4 & 10 \\
\hline XII & 2 & 4 & 2 & - & 2 & 10 \\
\hline Total & 21 & 23 & 31 & 5 & 21 & 101 \\
\hline
\end{tabular}

Fuente: Adaptado de Pires, Marques \& Castillo (2019)

Se puede observar en el cuadro 4 la distribución de los libros de minicursos por temas de matemáticas de las distribuidos en las tendencias de pesquisas en Historia de las Matemáticas. Del ciento y un (101) libros de minicursos, veintiún (21de éstos tratan asuntos de aritmética, más un porcentaje pequeños de estos, tratan tímida e implícitamente el tema de la geometría y 
el álgebra. Sin embargo, se caracterizaron como propios de la aritmética, ya que este tema es predominantemente explícito, y el objetivo del libro es tratar el tema de la aritmética.

Además, se determinó que veintitrés (23) libros de minicursos abordan temas relacionados con el álgebra. Es perceptible que hay una diferencia entre la cantidad de libros para aritmética y el álgebra más es relativamente pequeña. Las producciones que representan un número relativamente mayor en relación a los otros tópicos, son las que abordan temas propios de la geometría con un total de treinta y un (31) libros de Minicurso. Finalmente tenemos por un lado que le tópico menos abordado en las producciones es la trigonometría, con apenas cinco (05) libros y un restante de veinte (20) los cuales abordan temas que no se encajan con las primeras. Destacamos que recientemente fueron añadidos a esta colección los libros de Minicurso del XIII SNHM celebrado en Fortaleza (CE), Brasil, con lo cual se cuantifican en el CREPHIMat un total de 111 libros de minicursos (ver Figura 12) en el uso didáctico de la historia de la matemática por parte de los profesores en sus aulas de matemática.

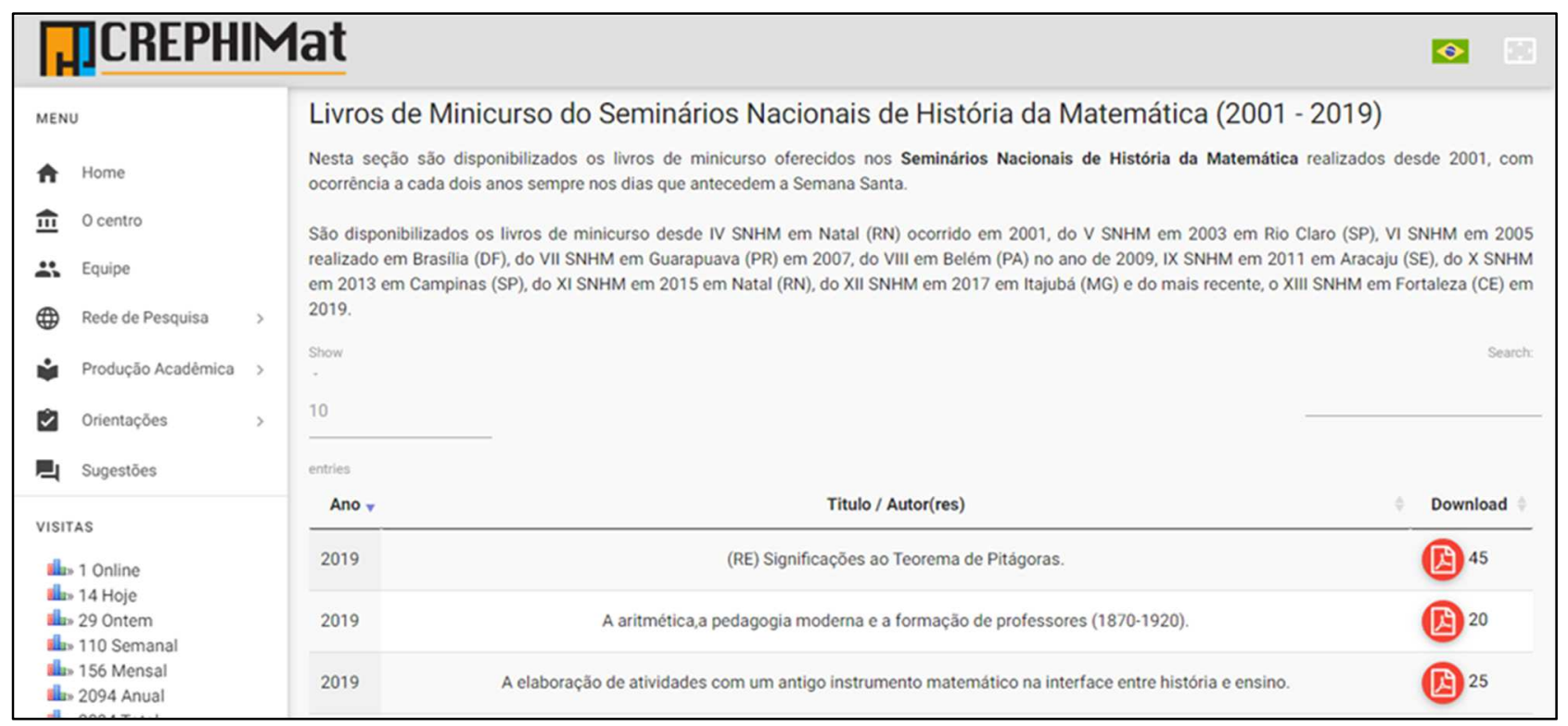

Figura 12. Libros de Minicurso del SNHM en el CREPHIMat

Fuente: Elaboración propia de los autores a partir de http://www.crephimat.com/livrosdeminicursos

El CREPHIMat también ofrece los Productos Educativos de Disertaciones de historia de la matemática oriundas de maestrías profesionales, tales producciones representan objetos de aprendizajes en forma de, por ejemplo, un pequeño libro, un manual, actividades, secuencia didáctica, software, juego educativo. Estos objetos de aprendizajes son desarrollados sobre la base de trabajos de investigación científica destinados a aportar contribuciones a la práctica profesional de profesores de Educación Básica, futuros profesores, profesores de Educación 
Superior y Formadores de profesores. En general, el producto presenta una propuesta para la enseñanza o para la formación del profesorado desarrollada por el maestrando y su tutor. En el siguiente cuadro 5 se presentan la cantidad de productos educativos catalogados y clasificados según las tres tendencias de pesquisas de la Historia de la matemática.

Cuadro 5: Productos Educativos según las tendencias de pesquisas

\begin{tabular}{|l|c|c|c|c|c|c|c|}
\hline & HEpM & \% & HEdM & \% & HEnM & \% & Total \\
\hline Productos Educativos & 1 & 1 & 32 & $46 \%$ & 36 & $53 \%$ & 69 \\
\hline
\end{tabular}

Fuente: Elaboración propia de los autores

Del cuadro 5 podemos observar que las dos tendencias de pesquisas en estos productos que más destacan son HEnM con el $53 \%$ de la cantidad total de los objetos de aprendizajes, seguida de la HEdM con un $46 \%$ de los productos y finalmente, la HEpM con apenas el 1\% restante. Estos productos en el CREPHIMat se presentan a la comunidad de la misma manera que las tesis y disertaciones, es decir, separadas en secciones según cada tendencia (ver Figura 13).

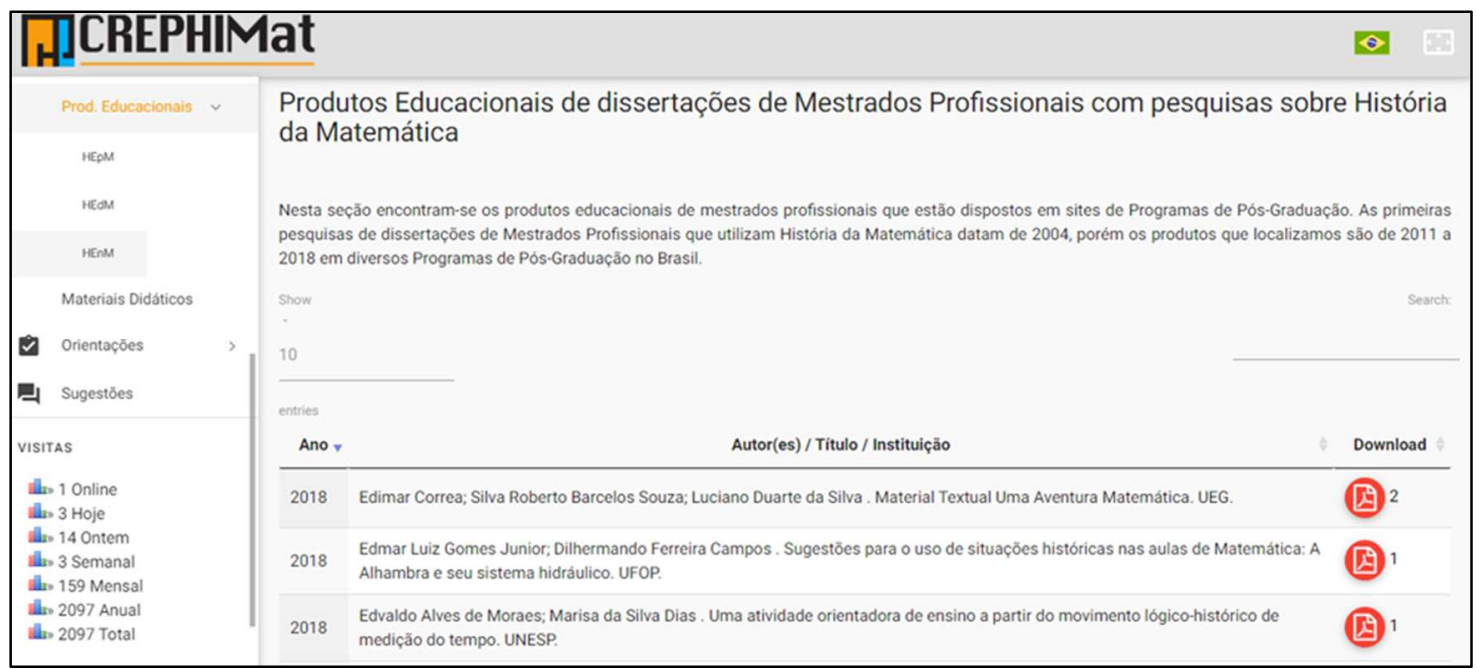

Figura 13. Productos educativos en el CREPHIMat

Fuente: Elaboración propia de los autores a partir de http://www.crephimat.com/pe3

Para terminar esta sección de producciones en el CREPHIMat disponibles principalmente para que los profesores, por un lado, realicen exploraciones didácticas e conceptuales de modo que puedan superar algunas dificultades teóricas y por el otro lado que puedan usar estos materiales diseñado en base de informaciones históricas, para apoyar sus acciones pedagógicas en sus aulas de matemática. La última sección, pero no menos importante 
fue denominada Materiais Didáticos, en el cual se depositan todas aquellas producciones que no se encajan en las anteriores categorías (Figura 14).

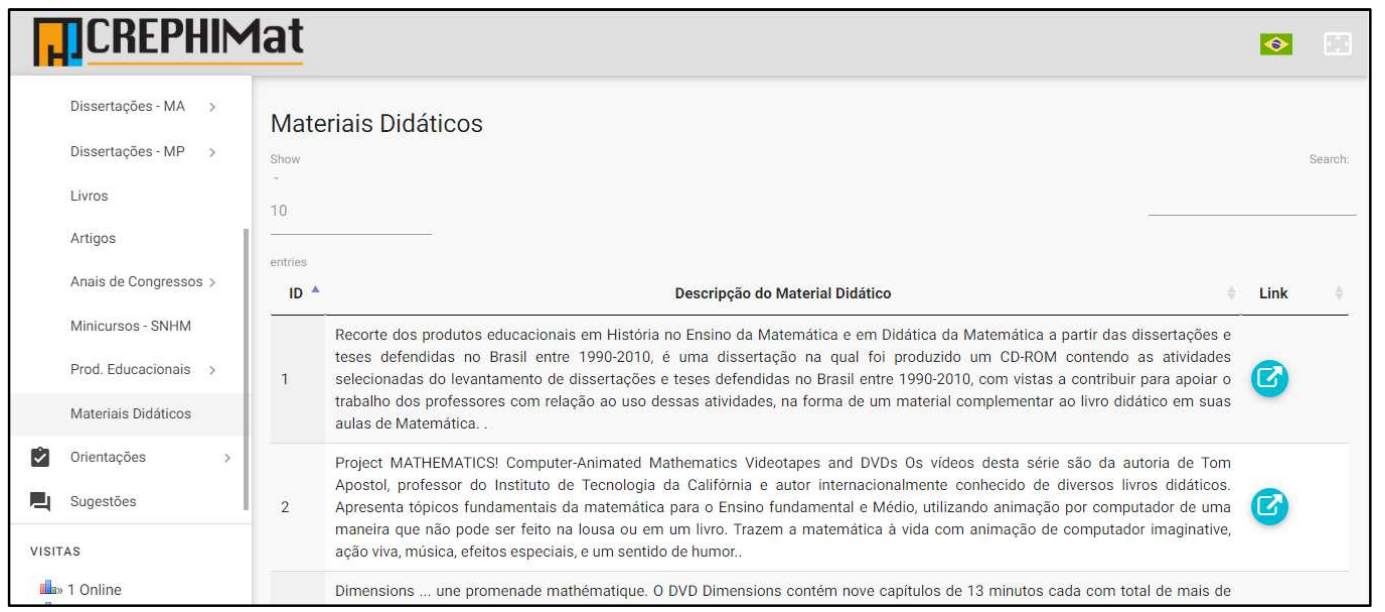

Figura 14. Materiales didácticos en el CREPHIMat

Fuente: Elaboración propia de los autores a partir de http://www.crephimat.com/materiais

\section{METRICAS Y AVALIACIÓN PARCIL DEL CREPHIMat}

El sábado 3 de agosto de 2019, fue cunado de manera oficial el Prof. Dr. Dr. Iran Abreu Mendes en un paso más de sus investigaciones financiadas por el CNPq, pone a disposición del público del área de Historia de las Matemáticas el Centro Brasileño de Referencia en Investigación en Historia de las Matemáticas (CREPHIMat), un Centro virtual que está poniendo a disposición toda la producción y otras informaciones sobre los estudios e investigaciones en el área. Desde ese punto de partida en conocimiento de nuestra comunidad el CREPHIMat ha recibido hasta el momento más de dos mil (2000) visitas (Figura 15) de estudiantes para profesor, profesores en ejercicios e investigadores del área.

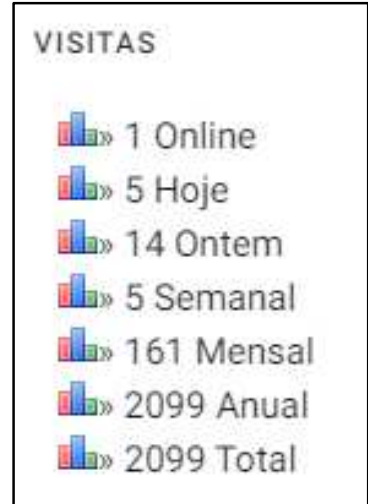

Figura 15. Métricas de visitas del CREPHIMat

Fuente: Elaboración propia de los autores 
Además de los cuantitativo de las visitas, en el CREPHIMat fue diseñado para cuantificar las visualizaciones/Download de algunas de sus producciones como Tesis, Disertaciones y Libros de minicursos. Con este cuantificador pudimos percibir por lo menos toda la colección de tesis contemplada en el centro ha tenido una media de 10 visualizaciones/Download, en cuanto a las disertaciones de maestrías académicas, ha conseguido una media de 7 visualizaciones/Download. Con respecto a las disertaciones de maestrías profesionales ha logrado una media de 5 visualizaciones/Download. En cuestión de los libros de minicursos de los SNHM, la media de toda la colección desde 2001- 2019 es de 12 visualizaciones/Download.

Además de estos cuantificadores que nos indican que el entorno virtual está siendo cada vez más accesado por nuestra comunidad académica con interés en la historia de la matemática. Además de esto, hemos recibido algunas evaluaciones por medio de un formulario online http://bit.ly/2qJ7fmU, con el tuvimos los siguientes contactos:

Un primer contacto lo realiza un estudiante de pregrado el cual nos comenta, primeramente: El CREPHIMat, fue decisivo para la realización de mis investigaciones para la construcción de un artículo y del trabajo de conclusión de grado TCC, ya que sólo en el CREPHIMat encontré todas las memorias del SNHM. Además, nos indica que la navegación por las secciones de CREPHIMat es Excelente, calificativa que también nos presenta para la organización de los contenidos en el centro virtual.

El siguiente contacto fue con un profesor el cual nos alertó con su mensaje: No puedo descargar los libros de los minicursos de la SNHM. ¿Puedes ver este problema? Co esto nos percatamos de algunos errores con los links de algunos libros de minicursos y pudimos ajustar para que el profesor pudiera tener acceso a estos materiales. Aunado a esto, el profesor nos indica que la navegación por las secciones de CREPHIMat es Excelente, y que la organización de los contenidos en el centro virtual en buena.

Un tercer contacto fue también por un profesor, que nos deja el siguiente mensaje en el área de Sugestões: ¡Felicito a los idealizados de este centro virtual por esta gran iniciativa, ya que es una idea innovadora y facilitadora para aquellos que desean encontrar producciones brasileñas en Historia de las Matemáticas!

El cuarto contacto fue por un pesquisador en el área de la matemática, el cual por la misma vía del formulario de Sugestões envía el siguiente mensaje: Me encantó el portal, siendo 
un investigador en Matemáticas y en fase de preparación de la tesis final para la maestría en educación, en Angola, las contribuciones registradas aquí me ayudarán mucho para mí el desarrollo de mi trabajo de grado y para mi desempeño profesional en el campo educativo.

Estos contactos nos dan evidencia de que el CREPHIMat podemos inferir que ha tenido un impacto positivo tanto para estudiante, profesores y pesquisadores interesados en el área de historia, tanto en Brasil como fuera del país, dado a que los primeros contactos fueron registrados desde Brasil, mientras que el último contacto fue desde Angola.

\section{CONSIDERACIONES FINALES}

Este articulo presentan resultados parciales de proyectos de pesquisas coordinados por el Prof. Dr. Iran Abreu Mendes, el primero intitulado: História para o Ensino da Matemática na Formação de Professores e na Educação Básica: uma Análise da Produção Brasileira (1997 - 2018) y el segundo intitulado: Uma história das pesquisas em História da Matemática no Brasil: produções, disseminações e contribuições à formação de professores de Matemática.

Estos resultados, por un lado, muestran la constitución de un gran acervo de producciones académico-científicas Brasil que traten sobre la historia de la matemática para el periodo de 1990-2018. Por el otro lado la materialización del Centro Brasileño de Referencia en Pesquisas sobre Historia de las Matemáticas - CREPHIMat. Destacando que este, cumpliendo una función como repositorio de contenidos digitales, actualmente posee por lo menos unos 2000 archivos, entre Tesis, Disertaciones, artículos de revistas, memorias de congresos, libros de minicursos, productos educativos, materiales didácticos y demás producciones dirigidos a estudiantes, profesores y pesquisadores interesados en la historia de las matemáticas.

Este trabajo caracteriza al CREPHIMat como un entorno virtual que permite centralizar la mayor cantidad de documentos digitales y/o digitalizados sobre la historia de las matemáticas, para ser preservados, difundíos y diseminados virtualmente al mayor número posible de personas interesadas en el debate y la producción sobre la historia de la matemática. De esta manera, con el CREPHIMat buscamos tanto contribuir con la comunidad de educadores matemáticos en general como de investigadores en Historia de la Matemática y Educación Matemática. En este sentido se disponibilizan estas producciones para apoyar las practicas pedagógicas en la aulas de matemáticas como de servir de un ambiente que permita a los pesquisador tener en cuenta que se ha producido en el área de historia de la matemática en sus 
tres tendencias y que falta es lo que estas investigaciones han dejado como cuestiones abiertas para fomentar otras investigaciones inéditas.

\section{Agradecimientos}

El presente artículo fue realizado con el apoyo tanto de la Coordenação de Aperfeiçoamento de Pessoal de Nível Superior - Brasil (CAPES) - Código de Financiamiento 001, como del Conselho Nacional de Desenvolvimento Científico e Tecnológico - Brasil $(\mathrm{CNPq})$.

\section{Referencias}

Barros, R. J., \& Mendes, I. A. (2017). Dissertações e teses em História e Epistemologia da Matemática: contribuições para a abordagem da Geometria Espacial no Ensino Médio. Principia, (37), 139-150.

Barros, R. J., \& Mendes, I. A. (2019). Descrição dos conteúdos de ensino superior presentes nas teses em história e epistemologia da matemática (1990-2010). COCAR, 13(25), 399-420.

Castillo, L. A., \& Mendes, I. A. (2019). Mapeamento dos artigos sobre história da matemática nas revista brasileiras (1985-2018). I Simpósio nacional sobre o ensino e pesquisa da matemática no contexto da educação, ciência e tecnologia. Belém: SBEM-PA.

Costa, D. A., \& Arruda, J. P. (2012). Repositório institucional de fontes para a história da educação matemática na universidade federal de santa catarina. I Encontro Nacional de Pesquisa em História da Educação Matemática - I ENAPHEM, 1-10. Vitória da Conquista: UESB.

Costa, D. A., \& Valente, W. R. (2015). História da Educação Matemática e o uso de um repositório de conteúdo digital. São Paulo: Livraria da Física.

CREMM. (2006). Centro de Referência de Modelagem Matemática no Ensino. Recuperado de http://www.furb.br/cremm/portugues/index.php

Gonçalves, F. D., \& Mendes, I. A. (2015). História da Educação Matemática no Brasil: abordagens que emergem das dissertações e teses defendidas entre 1990 e 2010. XI Seminário Nacional de História da Matemática, 1-14. Natal: SBHMat.

Mendes, I. A. (2008). Uma radiografia dos textos publicados nos Anais dos SNHM. 11 Seminário Nacional de História da Ciência e Tecnologia, 1-11. Niterói: SBHC.

Mendes, I. A. (2010). Cartografias da produção em História da Matemática no Brasil: um estudo centrado nas dissertações e teses defendidas entre 1990-2010. Poyecto de Pesquisa. Universidade Federal do Rio Grande do Norte, Natal. 
Mendes, I. A. (2012). Pesquisas em história da Educação Matemática no Brasil em três dimensões. Quipu, 14(1), 69-92.

Mendes, I. A. (2015). História da matemática no ensino: Entre trajetórias profissionais epistemológicas e pesquisas (1a ed.). São Paulo: Livraria da Fisica/SBHMat.

Mendes, I. A. (2018a). História para o Ensino de Matemática na Formação de Professores e na Educação Básica: uma Análise da Produção Brasileira (1997 - 2017). Poyecto de Pesquisa. Universidade Federal do Pará, Belém.

Mendes, I. A. (2018b). Uma história das pesquisas em História da Matemática no Brasil: produções, disseminações e contribuições à Formação de Professores de Matemática. Poyecto de Pesquisa. Universidade Federal do Pará.

Mendes, I. A. (2019). Flashes e Imagens das Produções nas Pesquisas em História da Matemática no Brasil: um cenário tecido em três décadas. XII Ecnontro Paraense de Educaçõ Matemática. Belém: SBEM-PA.

Pires, L. S., Marques, R., \& Castillo, L. A. (2019). Classificação dos livros de minicursos dos SNHM entre 2001-2017. En M. Chaquiam \& A. C. C. Pereira (Eds.), XIII Seminário Nacional de História da Matemática (pp. 1356-1369). Fortaleza: SBHMat.

Silva, L. P., Silva-Neto, B. C., \& Castillo, L. A. (2019). História para o ensino da matemática nos livros de minicursos do SNHM (2001 a 2017) para os anos iniciais do ensino fundamental. En M. Chaquiam \& A. C. C. Pereira (Eds.), XIII Seminário Nacional de História da Matemática (pp. 1437-1449). Fortaleza: SBHMat.

Autores:

Luis Andrés Castillo Bracho Mestrado em Educação em Ciências e Matemáticas (Educação Matemática), pelo Programa de Pós-Graduação em Educação em Ciências e Matemáticas (PPGECM) da Universidade Federal do Pará (UFPA/Brasil). Graduação em Licenciatura em Educação menção Matemática e Física pela Universidade do Zulia/Venezuela (2016). Pesquisador nível A-1 no Programa de Estímulo à Pesquisa e Inovação da Venezuela (2015 - Atual). Integrante

da Associaçao "Aprender en Red” e do Grupo de Pesquisa sobre Práticas Socioculturais e Educação Matemática (GPSEM/UFPA). Atua em temas de pesquisa como: uso de tecnologias digitais no ensino da Matemática e da Física. Mais informações no Curriculo Lattes: http://lattes.cnpq.br/4358821746569093. ORCID: https://orcid.org/0000-0002-5174-9148. E-mail: luiscastleb@gmail.com

Iran Abreu Mendes

Bolsista Produtividade em Pesquisa Nível 1C do CNPq. Pós-doutorado em Educação Matemática pela UNESP/Rio Claro (2008). Doutorado em Educação pela Universidade 
Federal do Rio Grande do Norte (2001) e Mestrado em Educação pela Universidade Federal do Rio Grande do Norte (1997). Graduação em Licenciatura em Matemática pela Universidade Federal do Pará (1983). Atualmente é professor Titular do Instituto de Educação Matemática e Científica da Universidade Federal do Pará (IEMCI), e pesquisador do Programa de Pós-graduação em Educação em Ciências e Matemáticas. Tem experiência no ensino de Cálculo, Geometria Analítica e Euclidiana, História da Matemática, História da Educação Matemática, Didática da Matemática e Fundamentos Epistemológicos da Matemática. Desenvolve pesquisas sobre: Epistemologia da Matemática, História da Matemática, História da Educação Matemática, História para o Ensino de Matemática, Práticas Socioculturais e Educação Matemática, Diversidade Cultural e Educação Matemática. Líder do Grupo de Pesquisa sobre Práticas Socioculturais e Educação Matemática

(GPSEM/UFPA). Mais informacões no Currículo Lattes: http://lattes.cnpq.br/4490674057492872. ORCID: https://orcid.org/0000-0001-7910-1602. URL: http://www.iranmendes.com. E-mail: iamendes1@gmail.com 